\section{Autores}

Maria Olinda Nogueira Ávila ${ }^{1}$

Paulo Novis Rocha²

Dirce MariaTrevisan Zanetta ${ }^{3}$ Luis $\mathrm{Yu}^{3}$

Emmanuel de Almeida Burdmann ${ }^{3}$

${ }^{1}$ Hospital São Rafael.

${ }^{2}$ Universidade Federal da Bahia.

${ }^{3}$ Universidade de São Paulo.
Data de submissão: 12/02/2014. Data de aprovação: 01/03/2014.

Correspondência para: Maria Olinda Nogueira Ávila. Hospital São Rafael.

Av. São Rafael, no 2152, São Marcos. Salvador, BA, Brasil. CEP: 41253-196.

E-mail: olindaavila@ @otmail.com

DOI: 10.5935/0101-2800.20140054

\title{
Balanço hídrico, injúria renal aguda e mortalidade de pacientes em unidade de terapia intensiva
}

\author{
Water balance, acute kidney injury and mortality of intensive care \\ unit patients
}

\section{Resumo}

Injúria renal aguda (IRA) é uma síndrome de elevada incidência, associada a altas taxas de morbimortalidade. Sepse, grandes cirurgias e baixo débito cardíaco são as principais causas de IRA no mundo. Na maioria destas situações clínicas, a expansão volêmica é o elemento fundamental de prevenção e do manejo terapêutico da IRA, restaurando a perfusão periférica e atenuando a nefrotoxicidade de drogas. Ressuscitação volêmica precoce em pacientes sépticos está associada à prevenção de isquemia tecidual e à maior sobrevida. Contudo, a manutenção de estratégia liberal de infusão de fluidos após ressuscitação inicial pode causar balanços hídricos cumulativamente positivos, e este vem sendo associado a aumento de morbimortalidade em pacientes criticamente enfermos. Neste trabalho, revisamos os principais estudos que associam balanço hídrico positivo $(\mathrm{BH}+)$ e morbimortalidade em pacientes internados em Unidades de Terapia Intensiva (UTI). Sugerimos que $\mathrm{BH}+$ (não apenas o volume urinário) possa ser utilizado como possível biomarcador precoce de IRA nestes pacientes.

Palavras-chave: balanço hídrico; insuficiência renal aguda; mortalidade; unidades de terapia intensiva.

INJÚRIA RENAL AGUDA E EXPANSÃO VOLÊMICA: BALANÇO HÍDRICO POSITIVO $(\mathrm{BH}+)$ É BENÉFICO?

Injúria renal aguda (IRA) ocorre em aproximadamente $3 \%$ a $15 \%$ dos pacientes internados e pode afetar $30 \%$ a $50 \%$ dos pacientes alocados em unidades de terapia intensiva (UTI). ${ }^{1-5}$ A mortalidade geral da IRA hospitalar é aproximadamente $20 \%$, podendo ultrapassar $50 \%$ em pacientes

\section{Abstract}

Acute kidney injury (AKI) has a high hospital incidence and is associated to significant morbidity and mortality. Sepsis, major surgery and low cardiac output are the main cause of AKI worldwide. In the majority of these situations, volume expansion is part of both prevention and therapeutic management, restoring peripheral perfusion and attenuating drug nephrotoxicity. Early and aggressive volume resuscitation in septic patients halts tissue ischemia and is associated with higher survival. However, a liberal fluid infusion strategy after six hours can cause fluid overload. Fluid overload has been associated with morbidity and mortality increase in critically ill patients. Herein, we present a review of the main studies that assessed the effects of net fluid balance/fluid overload on the morbidity and mortality of critically ill patients. We suggest that positive water balance may be used as a potential early biomarker of AKI in these patients.

Keywords: acute kidney injury; intensive care unit; mortality; water balance. criticamente enfermos. ${ }^{1-3}$ Os pacientes que desenvolvem IRA intra-hospitalar têm maior risco de desenvolver doença renal crônica ${ }^{4,5}$ e apresentam mortalidade tardia mais elevada após alta. $\cdot^{6-10} \mathrm{~A}$ prevenção da IRA passa pela identificação das suas principais causas. No contexto clínico de pacientes internados em UTI, a IRA tem etiologia predominantemente multifatorial: isquêmica e/ou nefrotóxica. ${ }^{11}$ 
Sepse é a causa de mais de $50 \%$ dos casos de IRA em UTI no mundo, seguida por grandes cirurgias e baixo débito cardíaco. ${ }^{1-10} \mathrm{Em}$ todas estas situações, a expansão volêmica é o elemento fundamental de prevenção e do manejo terapêutico, pois contribui para a restauração da perfusão periférica e atenua a nefrotoxicidade de drogas. ${ }^{3,12-15}$ Rivers et al. ${ }^{14}$ demonstraram, em 2001, em estudo randomizado e controlado, que ressuscitação volêmica agressiva precoce, orientada por protocolo com metas definidas, diminuiu significativamente a mortalidade na sepse grave. Apesar de inúmeras críticas no decorrer destes últimos 13 anos após sua publicação, ${ }^{15}$ este trabalho influenciou de forma marcante o tratamento de pacientes sépticos, tendo sido adotado como recomendação fundamental em todas as publicações da Surviving Sepsis Campaign. ${ }^{16}$ Atualmente, três ensaios clínicos controlados e randomizados estão em andamento, visando reproduzir os achados de Rivers: um nos EUA (Protocolized Care for Early Septic Shock - ProCESS), outro na Austrália (Australasian Resuscitation In Sepsis Evaluation Randomized Controlled Trial - ARISE) e o terceiro na Inglaterra. Embora o trabalho do grupo de Rivers não tenha explorado o impacto da ressuscitação volêmica na prevenção de IRA, Lin et al. ${ }^{17}$ demonstraram, em estudo posterior, que a ressuscitação volêmica precoce, semelhante à utilizada no protocolo de Rivers, diminuiu a incidência de IRA (de 55\% para $39 \%, p=0,015)$ em pacientes com choque séptico.

Assim, ressuscitação volêmica adequada nas primeiras seis horas de atendimento hospitalar em pacientes sépticos parece estar associada à prevenção de isquemia tecidual (incluindo isquemia renal) e à maior sobrevida. A ocorrência de balanço hídrico positivo $(\mathrm{BH}+)$ por um curto período de tempo em pacientes submetidos a estes protocolos de ressuscitação volêmica pode ser o custo a ser pago para restauração da perfusão tecidual. Contudo, a manutenção de estratégia liberal de infusão de fluidos após as primeiras seis horas de atendimento pode causar $\mathrm{BH}$ sucessivamente positivos. De fato, esta conduta não foi recomendada por Rivers, ${ }^{14}$ porém, é situação comum na prática clínica atual, ${ }^{18} \mathrm{com}$ consequências deletérias ao paciente.

É possível também que o benefício de ressuscitação volêmica guiada por metas não se deva apenas à administração de volumes maiores de fluidos para pacientes que especificamente assim o necessitam, de acordo com parâmetros hemodinâmicos pré-estabelecidos, mas à precocidade e adequação desta medida. A administração de fluidos limitada até a otimização de parâmetros hemodinâmicos estabelecidos previamente poderia resultar na infusão de menor volume de líquidos, minimizando $\mathrm{BH}+{ }^{19}$ Tokarik et al. ${ }^{20}$ em estudo piloto com 21 pacientes queimados, mostraram que a determinação da resposta a fluidos com o uso do LiDCO (Lithium Dilution Cardiac Output), durante a ressuscitação, permitiu a administração de menor volume de cristaloides. $\mathrm{O}$ estudo randomizou dois grupos de pacientes queimados: o grupo 1, cuja ressuscitação se baseou nas medidas de variação de pressão sistólica (SPV) e variação da pressão de pulso (PPV) com o LiDCO e o grupo 2, cuja ressuscitação se baseou nas fórmulas de uso habitual (Brooke/Parkland modificadas) para expansão volêmica de queimados. O Grupo 1 recebeu significativamente menos cristaloides do que o grupo $2(5.090 \pm 680 \mathrm{ml} \mathrm{e} 7.820 \pm 1.050 \mathrm{ml}$ respectivamente; $p=0,04) \cdot{ }^{20}$ Assim, ressuscitação volêmica guiada por metas não implica obrigatoriamente em maiores infusões de líquido e $\mathrm{BH}$ excessivamente positivo. ${ }^{19,20}$

\section{BALANÇO HÍDRICO POSITIVO E MORBIMORTALIDADE NA UTI: BH+ É PREJUDICIAL?}

Pacientes com $\mathrm{BH}$ persistentemente positivo estão mais sujeitos a repercussões clínicas desfavoráveis como congestão hepática, edema de alças intestinais com íleo paralítico, má absorção, hipertensão intraabdominal/síndrome de compartimento abdominal, edema miocárdico com distúrbios de condução e disfunção diastólica, congestão pulmonar com piora na complacência e trocas gasosas, edema cerebral, edema renal e edema de tecidos periféricos com cicatrização inadequada de feridas e infecções (Quadro 1). ${ }^{21-25}$ Órgãos encapsulados, como o rim e o fígado, têm capacidade limitada para acomodar excesso de líquido, o que pode determinar aumento da sua pressão intersticial, com consequente comprometimento do seu fluxo sanguíneo e deterioração funcional. ${ }^{13,22} \mathrm{O}$ aumento da pressão intersticial renal em pacientes com $\mathrm{BH}+$ acumulado causa hipoperfusão arterial e queda da filtração glomerular. Além disso, o aumento da pressão intra-abdominal (PIA), da pressão venosa central (PVC) e da pressão venosa renal (PVR), em condições de sobrecarga hídrica, contribui substancialmente para a queda da filtração glomerular, como sugerem 
estudos experimentais. Pressão venosa renal acima de $30 \mathrm{mmHg}$ por 2 horas em rins porcinos intactos resulta em queda importante do fluxo plasmático renal e da taxa de filtração glomerular. ${ }^{26}$ A elevação da PVR limita o fluxo sanguíneo renal e a formação de urina mais intensamente do que a hipoperfusão arterial renal. ${ }^{26,27} \mathrm{Em}$ pacientes com síndrome cardiorrenal, com ou sem baixo débito cardíaco, a elevação da PVC, PIA e PVR pode contribuir para disfunção renal progressiva. ${ }^{27} \mathrm{E}$ em pacientes submetidos à cirurgia cardíaca eletiva, a presença de PVC alta é um forte preditor de IRA no pós-operatório, independente da presença de baixo débito cardíaco. ${ }^{27,28}$

Em condições fisiológicas, a PIA varia de subatmosférica a 0 mmHg. Uma prolongada elevação da PIA igual ou acima de $12 \mathrm{mmHg}$ define hipertensão intra-abdominal. Níveis de PIA entre 10 a $15 \mathrm{mmHg}$ causam redução da perfusão mesentérica, com isquemia, inflamação e mais edema, que, por sua vez, acentua a hipertensão intra-abdominal, fechando o círculo vicioso. Quando a PIA alcança $20 \mathrm{mmHg}$, aumentam as chances de repercussões clínicas sistêmicas, tais como alterações cardiovasculares (aumento da PVC, redução do retorno venoso e do débito cardíaco), renais (redução do fluxo plasmático renal, aumento da PVR, congestão microvascular renal, aumento da resistência vascular renal, elevação de catecolaminas, angiotensina II e citocinas inflamatórias e hipertensão da cápsula renal), pulmonares (redução da complacência da parede torácica, aumento da pressão intratorácica, elevação do espaço morto, hipercapnia e hipoxia) e cerebrais (aumento da pressão intracraniana e queda da pressão de perfusão cerebral), configurando-se a síndrome do compartimento abdominal. ${ }^{29,30}$ Primordialmente, a síndrome do compartimento abdominal era descrita em pacientes submetidos a cirurgias abdominais ou vítimas de trauma, entretanto, atualmente, é cada vez mais diagnosticada em pacientes submetidos à ressuscitação volêmica maciça, politransfundidos, hipotérmicos ou coagulopatas, ou seja, pacientes com $\mathrm{BH}+$ sucessivos. Há evidências de que hipertensão intra-abdominal presente à admissão na UTI está associada a maior risco de disfunção orgânica grave (incluindo IRA) e a maior mortalidade. ${ }^{29,30}$

Outro aspecto não menos importante, que pode contribuir para os desfechos desfavoráveis associados ao $\mathrm{BH}+$, é o tipo de solução utilizada para ressuscitação volêmica. Coloides sintéticos podem ser potencialmente nefrotóxicos e estão associados a maior mortalidade, especialmente as soluções HES (bydroxyethyl starch) e possivelmente as gelatinas. ${ }^{31-33}$ Albumina 4\% deve ser evitada na ressuscitação volêmica de pacientes com traumatismo craniano, mas parece ser segura em pacientes sépticos com IRA ou em risco de desenvolvê-la. ${ }^{31-33} \mathrm{O}$ uso de albumina $20 \%$ vem sendo associado a menor incidência de IRA e redução de mortalidade em cirróticos com peritonite bacteriana espontânea, mas o seu uso rotineiro acaba limitado pelo maior custo em relações aos cristaloides. ${ }^{34}$ Desta forma, o Kidney Disease Improving Global Outcome (KDIGO) Clinical Practice Guideline para IRA, na sua mais recente edição, sugere que cristaloides isotônicos sejam preferíveis, ao invés dos coloides sintéticos ou não sintéticos, para expansão volêmica em pacientes sob risco de IRA ou com IRA instalada, na ausência de choque hemorrágico. ${ }^{32}$

Entretanto, os cristaloides não são isentos de risco. Estudos clínicos e experimentais sugerem que cristaloides não balanceados ou com maior conteúdo de cloro podem piorar acidose metabólica, causar vasoconstriç̧ão renal e reduzir o consumo renal de oxigênio. ${ }^{35-37}$ Chowdhury et al. ${ }^{36}$ realizaram um estudo de crossover em voluntários sadios, randomizados para receber ora 2 litros de salina

\begin{tabular}{|c|c|}
\hline QUADRO 1 & FEITOS DELETÉRIOS DO BALANÇO HÍDRICO POSITIVO (BH+) \\
\hline Local do Edema & Consequências \\
\hline Cérebro & Alterações cognitivas, delirium. \\
\hline Miocárdio & Alteração de contratilidade, disfunção diastólica, distúrbios de condução. \\
\hline Pulmões & Alterações de trocas gasosas, redução de complacência, aumento do trabalho respiratório. \\
\hline Fígado & Função sintética comprometida e colestase. \\
\hline Rins & Redução do fluxo plasmático renal e da taxa de filtração glomerular, retenção hidrossalina. \\
\hline Intestinos & Má absorção e íleo, culminando com síndrome de compartimento abdominal. \\
\hline Tecídos Periféricos & $\begin{array}{l}\text { Drenagem linfática comprometida, alterações da microcirculação, má cicatrização de feridas, } \\
\text { infecção de parede e úlceras de pressão. }\end{array}$ \\
\hline
\end{tabular}


isotônica $0,9 \%$ (que contém $150 \mathrm{mmol} / \mathrm{l}$ de cloro) ora 2 litros de plasmalyte (que contém $98 \mathrm{mmol} / \mathrm{l}$ de cloro) intravenosos. Os indivíduos, ao receberem salina isotônica $0,9 \%$, demoravam mais tempo para apresentar a primeira micção (de 90 para $142 \mathrm{~min}, p=$ $0,006)$, reduziam o volume urinário de $833 \mathrm{ml}$ para $523 \mathrm{ml}(p=0,002)$ e reduziam a velocidade do fluxo plasmático renal e a perfusão cortical renal, avaliados por tecnologia de ressonância magnética. ${ }^{36}$ Yunos et al. ${ }^{37}$ conduziram um estudo piloto, prospectivo, controlado, em pacientes criticamente enfermos, comparando a estratégia liberal de infusão de fluidos contendo cloro (como salina $0,9 \%$ e gelatinas), com o uso de soluções balanceadas ou com menor conteúdo de cloro, como solução de Hartmann (que contém $109 \mathrm{mmol} / \mathrm{l}$ de cloro) e plasmalyte. O manejo de fluidos com soluções balanceadas reduziu a frequência de hipercloremia, acidose metabólica e foi associada a significante redução na incidência de IRA e necessidade de terapia de suporte renal. ${ }^{37}$ Portanto, não somente a quantidade de fluido infundido na ressuscitação volêmica, mas a também a sua composição, pode interferir nos desfechos desfavoráveis associados ao $\mathrm{BH}+$.

Todas estas consequências deletérias do $\mathrm{BH}+$ tem sido associadas a maior mortalidade em pacientes criticamente enfermos. ${ }^{38-54}$ Nas Tabelas 1 e 2, estão resumidos os estudos mais importantes que se propuseram a avaliar o impacto do $\mathrm{BH}+$ na morbimortalidade de pacientes criticamente enfermos. ${ }^{38-54}$
Bouchard et al.$^{49}$ avaliaram a população adulta com IRA do estudo PICARD e observaram que, no momento do diagnóstico da IRA, o percentual de acúmulo de fluidos relativo ao peso da admissão na UTI foi menor em sobreviventes do que em não sobreviventes $(p=$ $0,01)$. Contudo, esta diferença não foi estatisticamente significante após ajuste para o escore APACHE III ( $p=$ $0,12)$. Quando o percentual de acúmulo de fluidos de todos os pacientes era superior a $10 \%$, caracterizando sobrecarga de líquido, a mortalidade em 30 e 60 dias se elevava de 25 para $37 \%(p=0,02)$ e de 35 para $48 \%$ $(p=0,01)$, respectivamente. $\mathrm{O}$ risco de morte associado à sobrecarga de líquido em pacientes dialisados foi de 2,07 (IC 95\% 1,27-3,37), ajustado pela gravidade da doença e pela modalidade de diálise. Nos pacientes não dialisados com sobrecarga de líquido, o risco de morte foi de 3,14 (IC 95\% 1,18-8,33). Os pacientes que mantiveram acúmulo de líquidos durante a hospitalização apresentaram maior mortalidade, que foi proporcional ao acúmulo de fluidos $(p<0,0001)$. Os pacientes nos quais a diálise foi capaz de corrigir a sobrecarga de líquido apresentaram mortalidade menor do que aqueles que mantiveram sobrecarga hídrica após diálise ( $35 \%$ x $56 \%$ respectivamente, $p=0,002)$. $\mathrm{O}$ risco de morte ajustado, associado à sobrecarga de líquido ao final da diálise foi 2,52 (IC 95\% 1,55-4,08). Os pacientes com sobrecarga hídrica no momento em que a creatinina estava mais elevada tiveram menor chance de recuperação da função renal $(35 \%$ x $52 \%$ respectivamente, $p=0,007)$. Este último achado sugere

\begin{tabular}{|c|c|c|c|c|c|c|c|c|}
\hline ABELA 1 & $\begin{array}{l}\text { JDOS AVALIAN } \\
\text { PIA INTENSIV }\end{array}$ & TI) & Al) & DRICU IL & vit & DE & $\begin{array}{c}D \\
\end{array}$ & 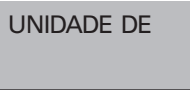 \\
\hline Estudo & Desenho & $\begin{array}{l}\text { População } \\
\text { e número }\end{array}$ & $\mathrm{n}$ & $\begin{array}{c}\text { BH menos } \\
\text { positivo }\end{array}$ & $\begin{array}{l}\text { BH mais } \\
\text { positivo }\end{array}$ & $\begin{array}{l}\text { Critério de } \\
\text { IRA }\end{array}$ & $\begin{array}{c}\text { Impacto na } \\
\text { função renal do } \\
\text { grupo menos } \\
\text { positivo }\end{array}$ & $\begin{array}{c}\text { Desfecho } \\
\text { principal do } \\
\text { grupo menos } \\
\text { positivo }\end{array}$ \\
\hline $\begin{array}{l}\text { Wiedemann, } \\
2006^{38}\end{array}$ & $\begin{array}{c}\text { ERC } \\
\text { multicêntrico }\end{array}$ & SARA & 1000 & -136 (7 dias) & $\begin{array}{l}+6992 \\
\text { (7 dias) }\end{array}$ & $\begin{array}{c}\text { Terapia } \\
\text { Suporte } \\
\text { Renal }\end{array}$ & Sem diferença & $\begin{array}{c}\text { Duração < VM } \\
\text { e UTI }\end{array}$ \\
\hline Martin $2005^{39}$ & ERC 1 Centro & ALI & 40 & $\begin{array}{l}-5480 \\
\text { (5 dias) }\end{array}$ & $\begin{array}{l}-1490 \\
(5 \text { dias })\end{array}$ & $\begin{array}{l}\text { Aumento } \\
\text { creatinina }\end{array}$ & Sem diferença & $\begin{array}{c}\text { Melhora } \\
\text { oxigenação }\end{array}$ \\
\hline Mitchel $1992^{40}$ & ERC 1 Centro & UTI mista & 102 & $+142 \mathrm{ml}$ & +2239 ml & $\begin{array}{l}\text { Aumento } \\
\text { creatinina }\end{array}$ & Discreta piora & $\begin{array}{c}\text { Duração < VM } \\
\text { e UTI }\end{array}$ \\
\hline Adesanya $2008^{43}$ & $\begin{array}{l}\text { Retrospectivo } \\
\text { Observacional }\end{array}$ & UTI cirúrgica & 41 & $+5 \mathrm{~kg}$ & $+8,3 \mathrm{~kg}$ & $\begin{array}{l}\text { Aumento } \\
\text { creatinina }\end{array}$ & Sem diferença & $\begin{array}{c}\text { Duração < VM } \\
\text { e UTI }\end{array}$ \\
\hline McArdle $2007^{44}$ & $\begin{array}{l}\text { Retrospectivo } \\
\text { Observacional }\end{array}$ & UTI cirúrgica & 100 & +7500 ml & $+10000 \mathrm{ml}$ & $\begin{array}{l}\text { Aumento } \\
\text { creatinina }\end{array}$ & Sem diferença & $\begin{array}{c}\text { complicação } \\
\text { pós-operatória }\end{array}$ \\
\hline Ariati $2007^{45}$ & Prospectivo & $\begin{array}{c}\text { UTI } \\
\text { queimado }\end{array}$ & 24 & +7500 ml & +12000 ml & Diurese & Sem diferença & DMOS \\
\hline
\end{tabular}

IRA: Injúria renal aguda; ERC: Ensaio randomizado e controlado; SARA: Síndrome da angústia respiratória do adulto; ALI: Lesão aguda pulmonar; VM: Ventilação mecânica; DMOS: Disfunção de múltiplos órgãos e sistemas. 
TABELA 2 ESTUdOS AVALIANDO IMPACTO DO BALANÇO HÍDRICO POSITIVO NA MORTALIDADE DE PACIENTES INTERNADOS EM UNIDADE DE TERAPIA INTENSIVA (UTI)

\begin{tabular}{|c|c|c|c|c|c|c|c|c|}
\hline \multirow{2}{*}{ Autor e ano } & \multirow{2}{*}{ Desenho } & \multirow{2}{*}{ População } & \multirow{2}{*}{$n$} & \multicolumn{2}{|c|}{$\mathrm{SH} \%$} & \multicolumn{2}{|c|}{$\mathrm{BH} \mathrm{ml} / 24$ horas } & \multirow{2}{*}{$p$} \\
\hline & & & & vivos & mortos & vivos & mortos & \\
\hline Goldstein, $2001^{46}$ & $\begin{array}{l}\text { Observacional } \\
\text { Retrospectivo }\end{array}$ & Pediátrica & 21 & 16 & 34 & - & - & 0,03 \\
\hline Goldstein $2005^{47}$ & $\begin{array}{c}\text { Observacional } \\
\text { Prospectivo }\end{array}$ & Pediátrica & 116 & 14 & 25 & - & - & $<0,03$ \\
\hline Foland $2004^{48}$ & $\begin{array}{l}\text { Observacional } \\
\text { Retrospectivo }\end{array}$ & Pediátrica & 113 & 8 & 15 & - & - & 0,02 \\
\hline Bouchard $2009^{49}$ & $\begin{array}{l}\text { Observacional } \\
\text { Retrospectivo }\end{array}$ & IRA & 396 & 9 & 14 & - & - & 0,01 \\
\hline Vaara ST $2012^{53}$ & $\begin{array}{c}\text { Observacional } \\
\text { Prospectivo } \\
\text { Multicêntrico }\end{array}$ & IRA & 283 & 31 & 59 & - & - & 0,03 \\
\hline Payen $2008^{51}$ & $\begin{array}{l}\text { Observacional } \\
\text { Retrospectivo }\end{array}$ & IRA & 1120 & - & - & $150^{*}$ & $980^{*}$ & $<0,001$ \\
\hline De Almeida $2012^{52}$ & $\begin{array}{c}\text { Observacional } \\
\text { Prospectivo }\end{array}$ & UTI Câncer & 122 & - & - & $887^{* *}$ & $1675^{* *}$ & 0,017 \\
\hline
\end{tabular}

SH: Sobrecarga hídrica ou ganho > 10\% do peso da admissão na UTI; BH ml/24 horas = média do balanço hídrico nas 24 horas; IRA: Injúria renal aguda. * Média do BH diário nos primeiros 3 dias de internação na UTI; ** Média do BH diário durante todo a internação na UTI.

que, após instalação da IRA, a administração de fluidos em excesso não exerce qualquer efeito protetor na recuperação renal. Entretanto, não é possível afirmar que a sobrecarga hídrica per se foi a causa do retardo da recuperação renal ou se os pacientes com sobrecarga hídrica já tinham IRA mais grave e, por isso, apresentaram recuperação mais tardia. ${ }^{37}$

Vaara ST et al., ${ }^{53}$ por meio de estudo multicêntrico prospectivo observacional com 296 pacientes de 17 UTIs, também relataram que sobrecarga hídrica (definida como acúmulo de fluidos acima de 10\% do peso admissional), no momento da indicação de terapia de suporte renal (TSR), foi associada à maior risco de morte em 90 dias (OR 2,6), após ajuste para gravidade da doença, tempo de início de TSR, modalidade de TSR e sepse. ${ }^{53}$ Embora a associação entre $\mathrm{BH}+$ e mortalidade pareça lógica e provável, a maioria dos trabalhos que a demonstram têm desenho observacional, não sendo capazes de estabelecer relação de causa e efeito definitiva. ${ }^{40,42,43,45-53}$ Nenhum destes estudos é capaz de esclarecer se pacientes com $\mathrm{BH}+$ tem maior mortalidade por serem mais graves (e por isso necessitam de maior aporte de drogas, antibióticos, sedativos, nutrição parenteral etc.) ou se o $\mathrm{BH}+$ realmente tem participação fisiopatológica independente no processo de morte. Alternativamente, podemos especular que o $\mathrm{BH}+$ seja um biomarcador precoce de IRA e, esta sim, esteja associada à mortalidade (Figura 1).
Figura 1. Possíveis associações entre injúria renal aguda (IRA), balanço hídrico positivo $(\mathrm{BH}+)$ e mortalidade. $\mathrm{O} \mathrm{BH}+$ pode estar associado a maior mortalidade por diversas vias fisiopatológicas (setas azuis): por causar edema visceral e periférico, dificultando o funcionamento orgânico; por diluição de antibióticos hidrofílicos, reduzindo a eficácia dos mesmos, ou até por hemodiluição da creatinina sérica, retardando o diagnóstico de injúria renal aguda (IRA). Entretanto, o $\mathrm{BH}+$ é mais frequentemente encontrado em pacientes de maior gravidade clínica (seta laranja) e em pacientes com IRA (seta vermelha), ambas as situações já independentemente associadas a maior mortalidade.

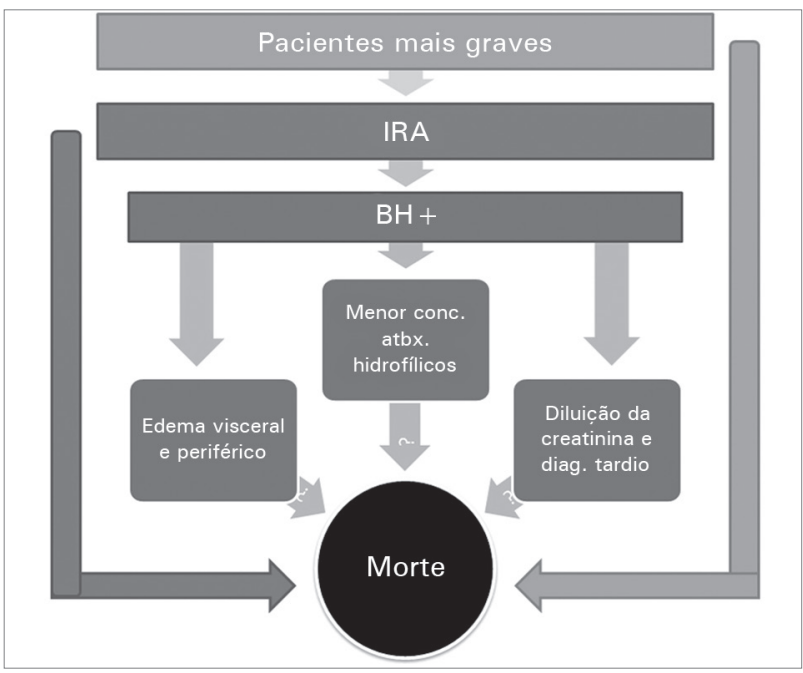

Existem poucos ensaios clínicos randomizados que estudaram a associação entre $\mathrm{BH}+\mathrm{e}$ mortalidade. Wiedemann et al. ${ }^{38}$ randomizaram 1000 pacientes com síndrome da angústia respiratória do adulto (SARA) para duas estratégias de manejo de líquidos, uma conservadora e a outra liberal. Este é o maior estudo prospectivo, controlado, avaliando o impacto 
da sobrecarga hídrica na duração da ventilação mecânica e mortalidade em SARA. A estratégia conservadora de infusão de líquidos reduziu o tempo de ventilação mecânica, sem piora da função renal, entretanto, a mortalidade foi semelhante nos dois grupos. $^{38}$

O maior ensaio clínico randomizado, controlado, desenhado com o objetivo de avaliar o impacto de estratégias de ressuscitação volêmica na mortalidade foi desenvolvido em seis centros clínicos da África. ${ }^{54}$ Os autores estudaram crianças ( $<12$ anos de idade) sépticas com sinais de hipoperfusão periférica, que foram randomizadas para ressuscitação com bolus de salina ( $20 \mathrm{ml} / \mathrm{kg}$ IV em 1 horas), bolus de albumina (20 ml/kg IV em 1 horas) ou nenhum bolus (controle). Os três grupos recebiam igualmente 2,5 a $4 \mathrm{ml} / \mathrm{kg} / \mathrm{h}$ de fluidos de manutenção, transfusões sanguíneas se a hemoglobina estivesse abaixo de $5 \mathrm{~g} / \mathrm{dl}$ e antibióticos apropriados. Foram excluídas crianças com gastroenterite, desnutrição grave e choque de causa não infecciosa. Crianças com hipotensão grave eram randomizadas para bolus de salina ou albumina, sem grupo controle. A mortalidade de 48 horas foi mais elevada nos grupos que receberam bolus intravenoso de salina $0,9 \%$ ou albumina, comparados ao grupo que não recebeu bolus $(11 \%$ ambos os grupos bolus versus $7 \%$; RR 1,$45 ; p=0,003) .{ }^{54}$ Não foram mostrados dados de $\mathrm{BH}$ de cada grupo. Além disso, o estudo foi conduzido em população pediátrica e em centros de saúde sem suporte avançado de vida, tornando difícil a generalização destes resultados para pacientes adultos internados em unidades de terapia intensiva.

Os efeitos mórbidos da sobrecarga hídrica nos diversos órgãos podem ser responsáveis pela associação entre $\mathrm{BH}+$ e mortalidade. Contudo, outro aspecto que também poderia contribuir para maior mortalidade dos pacientes com sobrecarga hídrica é a redução das concentrações plasmáticas de antibióticos hidrofílicos, por aumento do volume de distribuição dos mesmos. ${ }^{55}$ Como será discutido no tópico abaixo, sabe-se que pacientes inflamados/sépticos desenvolvem dano endotelial, com aumento da permeabilidade capilar predispondo a desvios de fluidos do intravascular para o interstício. Antibióticos hidrofílicos como aminoglicosídeos, beta-lactâmicos e glicopeptídeos poderão acompanhar este desvio de fluidos, resultando em concentrações plasmáticas subterapêuticas. ${ }^{55}$ Assim, a complexa associação entre $\mathrm{BH}+\mathrm{e}$ mortalidade ainda não está claramente elucidada. $\mathrm{O} \mathrm{BH}+$ seria um marcador de gravidade cardio/renal/microvascular ou é fator independente para maior mortalidade nos pacientes graves (Figura 1)?

\section{BALANÇO HÍDRICO POSITIVO E DIAGNÓSTICO DE IRA: BH+ INTERFERE COM O DIAGNÓSTICO DE IRA?}

Atualmente, o diagnóstico da IRA se fundamenta nos critérios RIFLE (Risk, Injury, Failure, Loss, Endstage Kidney Disease), AKIN (Acute Kidney Injury Network) e KDIGO (Kidney Disease Improving Global Outcomes), que são baseados em aumento da creatinina sérica e reduções do volume urinário. ${ }^{56,57}$ Estes critérios foram construídos a partir da observação de que pequenas elevações na creatinina sérica ${ }^{57}$ e reduções na diurese ${ }^{58}$ implicam em pior prognóstico para os pacientes. Os três sistemas de classificação são divididos em estágios de gravidade e há evidência de correlação crescente entre o estágio da IRA pelos critérios RIFLE e AKIN e a mortalidade. ${ }^{59} \mathrm{O}$ balanço hídrico não foi contemplado no diagnóstico de IRA por nenhum destes critérios atualmente adotados, fato que nos remete a uma reflexão sobre a fisiologia renal.

Sabe-se que um paciente com função renal normal e dieta usual (gerando $800 \mathrm{mOsm}$ de excretas por dia) é capaz de eliminar até 16 litros de urina maximamente diluída $(50 \mathrm{mOsm} / \mathrm{L})$, por supressão fisiológica do hormônio antidiurético (ADH). ${ }^{60}$ Por sua vez, quando um paciente criticamente enfermo desenvolve IRA diagnosticada pelos critérios habituais e recebe grandes infusões de fluidos (antibióticos, sedação, drogas vasoativas etc.), é fácil entender porque evolui com $\mathrm{BH}+$, uma vez que há evidente comprometimento na sua capacidade de excretar a sobrecarga hidrossalina recebida. Entretanto, pacientes criticamente enfermos sem o diagnóstico de IRA pelos critérios RIFLE, AKIN ou KDIGO também evoluem com BH positivo. Por que eles não conseguiriam aumentar adequadamente a excreção de sal e água livre, em resposta à sobrecarga hidrossalina?

Existem condições clínicas em UTI que podem predispor a $\mathrm{BH}+$, até mesmo em pacientes sem IRA. Hipotensão, baixo débito cardíaco, vasodilatação da sepse (ainda que sem hipotensão), hipoalbuminemia, ventilação mecânica $(\mathrm{VM})$ são capazes de ativar o sistema neuroendócrino (hormônio antidiurético, sistema nervoso simpático, sistema reninaangiotensina-aldosterona), com retenção tubular 
de água livre e sódio. ${ }^{12,13}$ Como estes pacientes, geralmente, recebem grande aporte hidrossalino, o resultado é $\mathrm{BH}$ positivo e edemas. VM com pressão positiva também pode reduzir a drenagem linfática, contribuindo para a formação de edema. ${ }^{12-14,25}$

Outros mecanismos fisiopatológicos que mediariam o desenvolvimento de $\mathrm{BH}+$ em pacientes criticamente enfermos sem o diagnóstico de IRA pelos critérios atuais envolvem, possivelmente, dois sistemas moleculares: o sistema angiopoietina/Tie (Ang/Tie) $)^{61,62}$ e o glicocálix endotelial. ${ }^{63,64} \mathrm{O}$ sistema angiopoietina/Tie (Ang/Tie) participa das principais funções do endotélio: angiogênese, manutenção da homeostase de fluidos, eletrólitos e transporte de proteínas pelas células endoteliais e inflamação/ coagulação desencadeados pela lesão endotelial. Estas três funções do endotélio estão alteradas na síndrome de disfunção orgânica múltipla (SDOM): a regulação do fluxo sanguíneo é alterada, a permeabilidade vascular se torna elevada com extravasamento de fluidos e células para os tecidos circundantes e os mecanismos de inflamação e coagulação são ativados. Estudos experimentais e clínicos sugerem que o sistema Ang/Tie participa da disfunção da barreira vascular observada em pacientes criticamente enfermos. ${ }^{61,62}$ A angiopoietina 1 (Ang-1), uma glicoproteína produzida constitutivamente pelos pericitos, causa estabilidade vascular ao se ligar ao receptor Tie-2 transmembrana das células endoteliais. Ao contrário, a angiopoietina 2 (Ang-2), liberada pelos corpúsculos de Weibel-Palade do citoplasma das células endoteliais por estímulos inflamatórios, antagoniza o efeito estabilizador da Ang-1, ligando-se competitivamente ao mesmo receptor Tie-2, fragilizando as junções intercelulares, predispondo a vazamento de fluidos, à inflamação e à coagulação. Pacientes com choque séptico apresentam níveis plasmáticos de Ang-2 elevados, que se correlacionam com $\mathrm{BH}+$, disfunção pulmonar e mortalidade. ${ }^{62}$

É possível também que o glicocálix endotelial esteja reduzido em situações de isquemia e/ou inflamação, como em sepse ou na síndrome de resposta inflamatória sistêmica (SRIS) ${ }^{63} \mathrm{O}$ glicocálix é uma estrutura formada de glicosaminoglicans e proteoglicans, que cobre a superfície endotelial voltada para a luz capilar, formando uma barreira contra a passagem de macromoléculas e limitando a adesão de células inflamatórias. A redução da sua espessura, em situações de isquemia/inflamação, determina aumento na permeabilidade vascular e adesão leucocitária, contribuindo para extravasamento de líquido e macromoléculas para o interstício. ${ }^{63,64}$ Portanto, agressões isquêmicas e inflamatórias, tão frequentes em pacientes criticamente enfermos, causam disfunção endotelial mediada por alterações no sistema angiopoietina $2 /$ Tie-2 e desnudamento no glicocálix endotelial, contribuindo para o desvio de líquido e de macromoléculas do compartimento intravascular para o interstício e, novamente, ativando o sistema neuroendócrino para reter fluidos. ${ }^{63,64}$

Por outro lado, pacientes criticamente enfermos podem desenvolver, isoladamente ou em conjunção aos eventos descritos acima, IRA precoce e sutil, que se manifesta pela incapacidade de controlar adequadamente o equilíbrio hidrossalino. As capacidades de concentração e diluição urinária dependem da integridade funcional da microvasculatura medular renal e podem estar prejudicadas em estágios muito precoces da doença renal. Enquanto a microcirculação cortical é capaz de fazer sua autorregulação por meio das mudanças de tônus das arteríolas aferente e eferente até determinados limites de pressão arterial média (PAM), a microcirculação medular parece ser mais dependente da PAM e da pressão de perfusão renal, sendo mais susceptível a isquemia e disfunção endotelial em situações de choque circulatório. ${ }^{65}$ Assim, $\mathrm{BH}$ positivo pode potencialmente ser biomarcador de IRA, antecedendo a elevação da creatinina ou a diminuição da diurese. Além disso, a sobrecarga de líquido pode causar hemodiluição e subestimar a medida do nível sérico da creatinina, retardando o diagnóstico de IRA pelos critérios usuais. ${ }^{18}$ Macedo et al., analisando dados do estudo PICARD, propuseram uso da seguinte fórmula para ajuste da creatinina sérica para o $\mathrm{BH}+$ :

Creatinina ajustada $=$ creatinina dosada $\times$ fator de correção ${ }^{*}$ * fator de correção $=(($ peso admissional $\times 0,6)+\Sigma \mathrm{BH}$ acumulado diário)/(peso admissional x 0,6).

O uso da creatinina ajustada por este "fator de correção" permitiu antecipar o diagnóstico de IRA em, no mínimo, um dia. ${ }^{18}$

Este conjunto de fatos sugere ser necessário ponderar a possível inclusão do $\mathrm{BH}+$ como critério de IRA, além da creatinina e do volume urinário. Três estudos observacionais, um retrospectivo com 90 pacientes, ${ }^{66}$ outro prospectivo com 100 pacientes ${ }^{67}$ e o último uma análise secundária de estudo prospectivo em 98 crianças 
sugeriram que $\mathrm{BH}+$ é marcador precoce de disfunção renal. Todos os estudos avaliaram pacientes submetidos à cirurgia cardiovascular ${ }^{65,66}$ Dass et al. ${ }^{66}$ realizaram análise post-hoc do Nesiritide Study, um ensaio clínico randomizado, placebo-controlado, de um único centro, desenhado para avaliar o impacto do uso profilático do nesiritide na necessidade de terapia de suporte renal e/ou na mortalidade de pacientes submetidos a cirurgia cardiovascular. Estes autores investigaram a hipótese de que o $\mathrm{BH}+$ nas primeiras 24 horas após a cirurgia cardiovascular poderia ser indicador de IRA no pós-operatório. Neste estudo, a incidência de IRA em pacientes com mediana de $\mathrm{BH}$ maior que $+849 \mathrm{ml} / 24$ horas foi de $80 \%$, versus $25 \%$ naqueles pacientes com mediana de $\mathrm{BH}-1221 \mathrm{ml} / 24$ horas $(p=0,001)$.

Kambhampati et al., ${ }^{67}$ em estudo observacional prospectivo, dividiram os grupos de $\mathrm{BH}$ em quartis, do intraoperatório até 48 horas de pós-operatório. A incidência de IRA no $4^{\circ}$ quartil (mediana de $\mathrm{BH}+$ $5000 \mathrm{ml} / 24$ horas) foi $52 \%$, em contraste com $16 \%$ de incidência de IRA no $1^{\circ}$ quartil (mediana de $\mathrm{BH}+500$ $\mathrm{ml} / 24$ horas), $p=0,016$. Análise multivariada ajustada para variáveis de confusão como idade, diabetes, HAS, tempo de circulação extracorpórea acima de $200 \mathrm{~min}$, tipo de cirurgia e taxa de filtração glomerular basal revelou que o quartil de $\mathrm{BH}+$ mais elevado (4을 quartil) se associou a risco significativamente maior de IRA (OR 4,89, IC 95\% 1,38-24,1; $p=0,046){ }^{67}$

$\mathrm{O}$ terceiro e mais recente estudo avaliou se a sobrecarga de fluidos no pós-operatório precoce de cirurgia cardíaca em 98 crianças estaria associada a maior morbidade, incluindo maior incidência de IRA. ${ }^{68}$ Sobrecarga de fluidos no pós-operatório precoce foi definida como $\mathrm{BH}+$ maior que $5 \%$ do peso basal, desde o pós-operatório imediato na UTI até o primeiro dia pós-operatório. Cinquenta por cento das crianças com sobrecarga hídrica na admissão da UTI desenvolveram IRA no primeiro dia pós-operatório, enquanto apenas $14,4 \%$ daquelas que não apresentaram sobrecarga hídrica precoce evoluíram com IRA $(p=0,023)$. A sobrecarga hídrica precedeu o aparecimento da IRA, ocorrida em qualquer dia pós-operatório. Sobrecarga hídrica de 8\% (o equivalente a $80 \mathrm{ml} / \mathrm{kg}$ ) mostrou uma especificidade de $90 \%$ para desenvolvimento subsequente de IRA RIFLE I ou F. A sobrecarga hídrica também foi associada a maior permanência hospitalar (3,5 dias a mais), dois dias a mais sob uso de inotrópicos e maior prevalência de VM prolongada. ${ }^{68}$ Até o momento, não existem evidências disponíveis testando a hipótese de que $\mathrm{BH}+$ é marcador precoce de IRA também em pacientes não cirúrgicos.

\section{SUMÁRIO}

Expansão volêmica precoce e guiada pela otimização de parâmetros de perfusão microcirculatória permanece como recomendação que reduz incidência de IRA e minimiza a mortalidade em pacientes sépticos. Expansão volêmica também é medida preventiva para IRA nefrotóxica, como na IRA induzida por contraste iodado, por aminoglicosídeos, anfotericina $\mathrm{B}$, rabdomiólise e síndrome de lise tumoral, dentre outras situações. Por sua vez, a manutenção de estratégias liberais de infusão de líquidos em pacientes criticamente enfermos, com ou sem IRA, pode levar a $\mathrm{BH}+$ acumulado e aumentar sua morbimortalidade. $\mathrm{BH}+$ pode também retardar o diagnóstico de IRA, por hemodiluição da creatinina sérica.

$\mathrm{BH}+$ poderia ser uma manifestação da incapacidade de controlar adequadamente o equilíbrio hidrossalino em pacientes criticamente enfermos e inflamados, por alterações na integridade funcional da microvasculatura medular renal.

Em conclusão, $\mathrm{BH}+$ pode ser biomarcador precoce de IRA e fator de risco independente para mortalidade em pacientes de UTI. São necessários ensaios clínicos randomizados para avaliar o complexo interrelacionamento entre $\mathrm{BH}+$, IRA e morte.

\section{ReferênCIAS}

1. Uchino S, Bellomo R, Morimatsu H, Morgera S, Schetz M, Tan I, et al. Continuous renal replacement therapy: a worldwide practice survey. The beginning and ending supportive therapy for the kidney (B.E.S.T. kidney) investigators. Intensive Care Med 2007;33:1563-70.

2. Fang Y, Ding X, Zhong Y, Zou J, Teng J, Tang Y, et al. Acute kidney injury in a Chinese hospitalized population. Blood Purif 2010;30:120-6. DOI: http://dx.doi.org/10.1159/000319972

3. Singbartl K, Kellum JA. AKI in the ICU: definition, epidemiology, risk stratification, and outcomes. Kidney Int 2012;81:819-25. PMID: 21975865 DOI: http://dx.doi.org/10.1038/ki.2011.339

4. Coca SG, Singanamala S, Parikh CR. Chronic kidney disease after acute kidney injury: a systematic review and meta-analysis. Kidney Int 2012;81:442-8. PMID: 22113526 DOI: http:// dx.doi.org/10.1038/ki.2011.379

5. Bucaloiu ID, Kirchner HL, Norfolk ER, Hartle JE $2^{\text {nd }}$, Perkins RM. Increased risk of death and de novo chronic kidney disease following reversible acute kidney injury. Kidney Int 2012;81:477-85. PMID: 22157656 DOI: http://dx.doi. org/10.1038/ki.2011.405

6. Lafrance JP, Miller DR. Acute kidney injury associates with increased long-term mortality. J Am Soc Nephrol 2010;21:34552. DOI: http://dx.doi.org/10.1681/ASN.2009060636 
7. Gammelager H, Christiansen CF, Johansen MB, Tønnesen E, Jespersen B, Sørensen HT. One-year mortality among Danish intensive care patients with acute kidney injury: a cohort study. Crit Care 2012;16:R124. DOI: http://dx.doi.org/10.1186/cc11420

8. Ponce D, Zorzenon Cde P, dos Santos NY, Balbi AL. Early nephrology consultation can have an impact on outcome of acute kidney injury patients. Nephrol Dial Transplant 2011;26:32026. DOI: http://dx.doi.org/10.1093/ndt/gfr359

9. Macedo E, Castro I, Yu L, Abdulkader RR, Vieira JM Jr. Impact of mild acute kidney injury (AKI) on outcome after open repair of aortic aneurysms. Ren Fail 2008;30:287-96. DOI: http://dx.doi.org/10.1080/08860220701857522

10. United States Renal Data System USRDS 2012 Annual Data Report: Atlas of CKD and ESRD in the United States. Am J Kidney Dis 2013;61:e1-e480.

11. Santos WJ, Zanetta DM, Pires AC, Lobo SM, Lima EQ, Burdmann EA. Patients with ischaemic, mixed and nephrotoxic acute tubular necrosis in the intensive care unit-a homogeneous population? Crit Care 2006;10:R68. DOI: http://dx.doi.org/10.1186/cc4904

12. Schrier R. Fluid administration in critically ill patients with acute kidney injury. Clin J Am Soc Nephrol 2010;5:733-9. DOI: http://dx.doi.org/10.2215/CJN.00060110

13. Townsend DR, Bagshaw SM. New insights on intravenous fluids, diuretics and acute kidney injury. Nephron Clin Pract 2008;109:c206-16. PMID: 18802369 DOI: http://dx.doi. org/10.1159/000142930

14. Rivers E, Nguyen B, Havstad S, Ressler J, Muzzin A, Knoblich B, et al. Early goal-directed therapy in the treatment of severe sepsis and septic shock. N Engl J Med 2001;345:1368-77. DOI: http://dx.doi.org/10.1056/NEJMoa010307

15. Venkataraman R, Kellum JA. Sepsis: update in the management. Adv Chronic Kidney Dis 2013;20:6-13. DOI: http:// dx.doi.org/10.1053/j.ackd.2012.10.013

16. Bion J, Jaeschke R, Thompson BT, Levy M, Dellinger RP. Surviving sepsis campaign: international guidelines for management of severe sepsis and septic shock: 2008. Intensive Care Med. 2008;34:11634. DOI: http://dx.doi.org/10.1007/s00134-008-1090-z

17. Lin SM, Huang CD, Lin HC, Liu CY, Wang CH, Kuo HP. A modified goal-directed protocol improves clinical outcomes in intensive care unit patients with septic shock: a randomized controlled trial. Shock 2006;26:551-7. PMID: 17117128 DOI: http://dx.doi.org/10.1097/01.shk.0000232271.09440.8f

18. Macedo E, Bouchard J, Soroko SH, Chertow GM, Himmelfarb J, Ikizler TA, et al. Fluid accumulation, recognition and staging of acute kidney injury in critically-ill patients. Crit Care 2010;14:R82. DOI: http://dx.doi.org/10.1186/cc9004

19. Prowle JR, Chua HR, Bagshaw SM, Bellomo R. Clinical review: Volume of fluid resuscitation and the incidence of acute kidney injury - a systematic review. Crit Care 2012;16:230. DOI: http://dx.doi.org/10.1186/cc11345

20. Tokarik M, Sjöberg F, Balik M, Pafcuga I, Broz L. Fluid therapy LiDCO controlled trial-optimization of volume resuscitation of extensively burned patients through noninvasive continuous real-time hemodynamic monitoring LiDCO. J Burn Care Res 2013;34:53742. DOI: http://dx.doi.org/10.1097/BCR.0b013e318278197e

21. Yohannes S, Chawla LS. Evolving practices in the management of acute kidney injury in the ICU (Intensive Care Unit). Clin Nephrol 2009;71:602-7. DOI: http://dx.doi.org/10.5414/CNP71602

22. Grams ME, Estrella MM, Coresh J, Brower RG, Liu KD; National Heart, Lung, and Blood Institute Acute Respiratory Distress Syndrome Network. Fluid balance, diuretic use, and mortality in acute kidney injury. Clin J Am Soc Nephrol 2011;6:966-73. DOI: http://dx.doi.org/10.2215/CJN.08781010

23. Prowle JR, Echeverri JE, Ligabo EV, Ronco C, Bellomo R. Fluid balance and acute kidney injury. Nat Rev Nephrol 2010;6:10715. DOI: http://dx.doi.org/10.1038/nrneph.2009.213

24. Bagshaw SM, Brophy PD, Cruz D, Ronco C. Fluid balance as a biomarker: impact of fluid overload on outcome in critically ill patients with acute kidney injury. Crit Care 2008;12:169. DOI: http://dx.doi.org/10.1186/cc6948
25. Bouchard J, Mehta RL. Fluid accumulation and acute kidney injury: consequence or cause. Curr Opin Crit Care 2009;15:509-13. DOI: http://dx.doi.org/10.1097/ MCC.0b013e328332f653

26. Doty JM, Saggi BH, Sugerman HJ, Blocher CR, Pin R, Fakhry $\mathrm{I}$, et al. Effect of increased renal venous pressure on renal function J Trauma 1999;47:1000-3. PMID: 10608524 DOI: http:// dx.doi.org/10.1097/00005373-199901000-00057

27. Lazzeri C, Valente S, Tarquini R, Gensini GF. Cardiorenal syndrome caused by heart failure with preserved ejection fraction Int J Nephrol 2011;2011:634903. PMID: 21331316 DOI: http://dx.doi.org/10.4061/2011/634903

28. Nohria A, Hasselblad V, Stebbins A, Pauly DF, Fonarow GC, Shah M, et al. Cardiorenal interactions: insights from the ESCAPE trial. J Am Coll Cardiol 2008;51:1268-74. DOI: http:// dx.doi.org/10.1016/j.jacc.2007.08.072

29. Dalfino L, Sicolo A, Paparella D, Mongelli M, Rubino G, Brienza N. Intra-abdominal hypertension in cardiac surgery. Interact Cardiovasc Thorac Surg 2013;17:644-51. DOI: http:// dx.doi.org/10.1093/icvts/ivt272

30. Cheatham ML, Malbrain ML, Kirkpatrick A, Sugrue M, Parr M, De Waele J, et al. Results from the International Conference of Experts on Intra-abdominal Hypertension and Abdominal Compartment Syndrome. II. Recommendations. Intensive Care Med 2007;33:951-62.

31. Prowle JR, Bellomo R. Fluid administration and the kidney. Curr Opin Crit Care 2013;19:308-14. DOI: http://dx.doi. org/10.1097/MCC.0b013e3283632e29

32. Kidney Disease Improving Global Outcomes (KDIGO) Clinical Practice Guideline for Acute Kidney Injury. Kidney Int Suppl. 2012;2:S1-138.

33. Severs D, Hoorn EJ, Rookmaaker MB. A critical appraisal of intravenous fluids: from the physiological basis to clinical evidence. Nephrol Dial Transplant 2014 Jan 23. [Epub ahead of print] DOI: http://dx.doi.org/10.1093/ndt/gfu005

34. Sort P, Navasa M, Arroyo V, Aldeguer X, Planas R, Ruiz-del-Arbol L, et al. Effect of intravenous albumin on renal impairment and mortality in patients with cirrhosis and spontaneous bacterial peritonitis. N Engl J Med 1999;341:4039. PMID: 10432325 DOI: http://dx.doi.org/10.1056/ NEJM199908053410603

35. Aksu U, Bezemer R, Yavuz B, Kandil A, Demirci C, Ince C. Balanced vs unbalanced crystalloid resuscitation in a near-fatal model of hemorrhagic shock and the effects on renal oxygenation, oxidative stress, and inflammation. Resuscitation 2012;83:767-73. DOI: http://dx.doi.org/10.1016/j.resuscitation.2011.11.022

36. Chowdhury AH, Cox EF, Francis ST, Lobo DN. A randomized, controlled, double-blind crossover study on the effects of 2-L infusions of $0.9 \%$ saline and plasma-lyte ${ }^{\circledR} 148$ on renal blood flow velocity and renal cortical tissue perfusion in healthy volunteers. Ann Surg 2012;256:18-24. PMID: 22580944 DOI: http://dx.doi.org/10.1097/SLA.0b013e318256be72

37. Yunos NM, Bellomo R, Hegarty C, Story D, Ho L, Bailey M. Association between a chloride-liberal vs chloride-restrictive intravenous fluid administration strategy and kidney injury in critically ill adults. JAMA 2012;308:1566-72. PMID: 23073953 DOI: http://dx.doi.org/10.1001/jama.2012.13356

38. National Heart, Lung, and Blood Institute Acute Respiratory Distress Syndrome (ARDS) Clinical Trials Network; Wiedemann HP, Wheeler AP, Bernard GR, Thompson BT, Hayden D, deBoisblanc B, et al. Comparison of two fluid-management strategies in acute lung injury. N Engl J Med 2006;354:256475. PMID: 16714767 DOI: http://dx.doi.org/10.1056/NEJMoa062200

39. Martin GS, Moss M, Wheeler AP, Mealer M, Morris JA, Bernard GR. A randomized, controlled trial of furosemide with or without albumin in hypoproteinemic patients with acute lung injury. Crit Care Med 2005;33:1681-7. PMID: 16096441 DOI: http://dx.doi.org/10.1097/01.CCM.0000171539.47006.02 
40. Mitchell JP, Schuller D, Calandrino FS, Schuster DP. Improved outcome based on fluid management in critically ill patients requiring pulmonary artery catheterization. Am Rev Respir Dis 1992;145:990-8. PMID: 1586077 DOI: http://dx.doi. org/10.1164/ajrccm/145.5.990

41. Brandstrup B, Tønnesen H, Beier-Holgersen R, Hjortsø E, Ørding H, Lindorff-Larsen K, et al. Effects of intravenous fluid restriction on postoperative complications: comparison of two perioperative fluid regimens: a randomized assessor-blinded multicenter trial. Ann Surg 2003;238:641-8. PMID: 14578723 DOI: http://dx.doi.org/10.1097/01.sla.0000094387.50865.23

42. Vidal MG, Ruiz Weisser J, Gonzalez F, Toro MA, Loudet C, Balasini $\mathrm{C}$, et al. Incidence and clinical effects of intra-abdominal hypertension in critically ill patients. Crit Care Med 2008;36:1823-31. DOI: http://dx.doi.org/10.1097/CCM.0b013e31817c7a4d

43. Adesanya A, Rosero E, Timaran C, Clagett P, Johnston WE. Intraoperative fluid restriction predicts improved outcomes in major vascular surgery. Vasc Endovascular Surg 2008;42:531-6. PMID: 18583299 DOI: http://dx.doi. org/10.1177/1538574408318474

44. McArdle GT, McAuley DF, McKinley A, Blair P, Hoper M, Harkin DW. Preliminary results of a prospective randomized trial of restrictive versus standard fluid regime in elective open abdominal aortic aneurysm repair. Ann Surg 2009;250:28-34. PMID: 19561485 DOI: http://dx.doi.org/10.1097/SLA.0b013e3181ad61c8

45. Arlati S, Storti E, Pradella V, Bucci L, Vitolo A, Pulici M. Decreased fluid volume to reduce organ damage: a new approach to burn shock resuscitation? A preliminary study. Resuscitation 2007;72:371-8. PMID: 17137702 DOI: http://dx.doi. org/10.1016/j.resuscitation.2006.07.010

46. Goldstein SL, Currier H, Graf Cd, Cosio CC, Brewer ED, Sachdeva R. Outcome in children receiving continuous venovenous hemofiltration. Pediatrics 2001;107:1309-12. DOI: http:// dx.doi.org/10.1542/peds.107.6.1309

47. Goldstein SL, Somers MJ, Baum MA, Symons JM, Brophy PD, Blowey D, et al. Pediatric patients with multi-organ dysfunction syndrome receiving continuous renal replacement therapy. Kidney Int 2005;67:653-8. PMID: 15673313 DOI: http:// dx.doi.org/10.1111/j.1523-1755.2005.67121.x

48. Foland JA, Fortenberry JD, Warshaw BL, Pettignano R, Merritt RK, Heard ML, et al. Fluid overload before continuous hemofiltration and survival in critically ill children: a retrospective analysis. Crit Care Med 2004;32:1771-6. DOI: http:// dx.doi.org/10.1097/01.CCM.0000132897.52737.49

49. Bouchard J, Soroko SB, Chertow GM, Himmelfarb J, Ikizler TA, Paganini EP, et al.; Program to Improve Care in Acute Renal Disease (PICARD) Study Group. Fluid accumulation, survival and recovery of kidney function in critically ill patients with acute kidney injury. Kidney Int 2009;76:422-7.

50. Vincent JL, Sakr Y, Sprung CL, Ranieri VM, Reinhart K, Gerlach H, et al.; Sepsis Occurrence in Acutely Ill Patients Investigators. Sepsis in European intensive care units: results of the SOAP study. Crit Care Med 2006;34:344-53. DOI: http:// dx.doi.org/10.1097/01.CCM.0000194725.48928.3A

51. Payen D, de Pont AC, Sakr Y, Spies C, Reinhart K, Vincent JL; Sepsis Occurrence in Acutely Ill Patients (SOAP) Investigators. A positive fluid balance is associated with a worse outcome in patients with acute renal failure. Crit Care 2008;12:R74. DOI: http://dx.doi.org/10.1186/cc6916

52. de Almeida JP, Palomba H, Galas FR, Fukushima JT, Duarte FA, Nagaoka D, et al. Positive fluid balance is associated with reduced survival in critically ill patients with cancer. Acta Anaesthesiol Scand 2012;56:712-7. DOI: http://dx.doi. org/10.1111/j.1399-6576.2012.02717.x

53. Vaara ST, Korhonen AM, Kaukonen KM, Nisula S, Inkinen O, Hoppu S, et al.; The FINNAKI study group. Fluid overload is associated with an increased risk for 90-day mortality in critically ill patients with renal replacement therapy: data from the prospective FINNAKI study. Crit Care 2012;16:R197. DOI: http://dx.doi.org/10.1186/cc11682
54. Maitland K, Kiguli S, Opoka RO, Engoru C, Olupot-Olupot P, Akech SO, et al.; FEAST Trial Group. Mortality after fluid bolus in African children with severe infection. $\mathrm{N}$ Engl J Med 2011;364:2483-95. PMID: 21615299 DOI: http://dx.doi. org/10.1056/NEJMoa1101549

55. Eyler RF, Mueller BA; Medscape. Antibiotic dosing in critically ill patients with acute kidney injury. Nat Rev Nephrol 2011;7:226-35. DOI: http://dx.doi.org/10.1038/nrneph.2011.12

56. Bellomo R, Ronco C, Kellum JA, Mehta RL, Palevsky P; Acute Dialysis Quality Initiative workgroup. Acute renal failure - definition, outcome measures, animal models, fluid therapy and information technology needs: the Second International Consensus Conference of the Acute Dialysis Quality Initiative (ADQI) Group. Crit Care 2004;8:R204-12.

57. Lassnigg A, Schmid ER, Hiesmayr M, Falk C, Druml W, Bauer P, et al. Impact of minimal increases in serum creatinine on outcome in patients after cardiothoracic surgery: do we have to revise current definitions of acute renal failure? Crit Care Med 2008;36:1129-37. DOI: http://dx.doi.org/10.1097/ CCM.0b013e318169181a

58. Avila MO, Zanetta DM, Abdulkader RC, Yu L, Burdmann EA. Urine volume in acute kidney injury: how much is enough? Ren Fail 2009;31:884-90.

59. Ricci Z, Cruz D, Ronco C. The RIFLE criteria and mortality in acute kidney injury: A systematic review. Kidney Int 2008;73:538-46. DOI: http://dx.doi.org/10.1038/ si.ki. 5002743

60. Bichet DG. Urine Output in Diabetes Insipidus. In: Sterns RH, Forman JP, eds. Up to Date; 2013 [Acessed May 18 2014]. Available from: http://www.uptodate.com/contents/ urine-output-in-diabetes-insipidus? source=search_result\&se arch=Urine+Output+in+Diabetes+Insipidus. \&selectedTitle= $1 \% 7 \mathrm{E} 150$

61. van Meurs M, Kümpers P, Ligtenberg JJ, Meertens JH, Molema G, Zijlstra JG. Bench-to-bedside review: Angiopoietin signalling in critical illness - a future target? Crit Care 2009;13:207. DOI: http://dx.doi.org/10.1186/cc7153

62. van der Heijden M, Pickkers P, van Nieuw Amerongen GP, van Hinsbergh VW, Bouw MP, van der Hoeven JG, et al. Circulating angiopoietin-2 levels in the course of septic shock: relation with fluid balance, pulmonary dysfunction and mortality. Intensive Care Med 2009;35:1567-74. DOI: http://dx.doi. org/10.1007/s00134-009-1560-y

63. De Backer D, Donadello K, Taccone FS, Ospina-Tascon G, Salgado D, Vincent JL. Microcirculatory alterations: potential mechanisms and implications for therapy. Ann Intensive Care 2011;1:27. DOI: http://dx.doi.org/10.1186/21105820-1-27

64. Mulivor AW, Lipowsky HH. Inflammation- and ischemia-induced shedding of venular glycocalyx. Am J Physiol Heart Circ Physiol 2004;286:H1672-80. PMID: 14704229 DOI: http:// dx.doi.org/10.1152/ajpheart.00832.2003

65. Chappell D, Jacob M, Hofmann-Kiefer K, Conzen P, Rehm M. A rational approach to perioperative fluid management. Anesthesiology 2008;109:723-40. PMID: 18813052 DOI: http:// dx.doi.org/10.1097/ALN.0b013e3181863117

66. Dass B, Shimada M, Kambhampati G, Ejaz NI, Arif AA, Ejaz AA. Fluid balance as an early indicator of acute kidney injury in CV surgery. Clin Nephrol 2012;77:438-44. PMID: 22595385 DOI: http://dx.doi.org/10.5414/CN107278

67. Kambhampati G, Ross EA, Alsabbagh MM, Asmar A, Pakkivenkata U, Ejaz NI, et al. Perioperative fluid balance and acute kidney injury. Clin Exp Nephrol 2012;16:730-8. DOI: http:// dx.doi.org/10.1007/s10157-012-0623-5

68. Hassinger AB, Wald EL, Goodman DM. Early postoperative fluid overload precedes acute kidney injury and is associated with higher morbidity in pediatric cardiac surgery patients. Pediatr Crit Care Med 2014;15:131-8. DOI: http://dx.doi. org/10.1097/PCC.0000000000000043 